\title{
Sensing Vehicle Dynamics for Determining Driver Phone Use
}

\author{
Yan Wang \\ Stevens Institute of \\ Technology \\ Hoboken, NJ 07030, USA \\ ywang48@stevens.edu \\ Yingying Chen \\ Stevens Institute of \\ Technology \\ Hoboken, NJ 07030, USA \\ yingying.chen@stevens.edu
}

\author{
Jie Yang \\ Oakland University \\ Rochester, MI 48309, USA \\ yang@oakland.edu \\ Marco Gruteser \\ Rutgers University \\ North Brunswick, NJ 08902, \\ USA
}

\author{
Hongbo Liu \\ Stevens Institute of \\ Technology \\ Hoboken, NJ 07030, USA \\ hliu3@stevens.edu \\ Richard P. Martin \\ Rutgers University \\ North Brunswick, NJ 08902, \\ USA \\ rmartin@rutgers.edu
}

\begin{abstract}
This paper utilizes smartphone sensing of vehicle dynamics to determine driver phone use, which can facilitate many traffic safety applications. Our system uses embedded sensors in smartphones, i.e., accelerometers and gyroscopes, to capture differences in centripetal acceleration due to vehicle dynamics. These differences combined with angular speed can determine whether the phone is on the left or right side of the vehicle. Our low infrastructure approach is flexible with different turn sizes and driving speeds. Extensive experiments conducted with two vehicles in two different cities demonstrate that our system is robust to real driving environments. Despite noisy sensor readings from smartphones, our approach can achieve a classification accuracy of over $90 \%$ with a false positive rate of a few percent. We also find that by combining sensing results in a few turns, we can achieve better accuracy (e.g., $95 \%$ ) with a lower false positive rate.
\end{abstract}

\section{Categories and Subject Descriptors}

H.4 [Information Systems]: Miscellaneous

\section{Keywords}

Driving Safety, Driver Phone Detection, Smartphone, Accelerometer, Gyroscope

\section{INTRODUCTION}

Distracted driving due to mobile devices contributes to nearly one thousand fatalities per year [4] and is now receiving attention not only from government regulators but also within the highest executive levels of the mobile industry [2]. Indeed, the National Transportation Safety Board has called for a nationwide ban on mobile devices behind the wheel [11], while the mobile industry has adopted a subtler approach with apps that seek to manage distraction. The AT\&T DriveSafe app [3], for example, silences the

Permission to make digital or hard copies of all or part of this work for personal or classroom use is granted without fee provided that copies are not made or distributed for profit or commercial advantage and that copies bear this notice and the full citation on the first page. To copy otherwise, to republish, to post on servers or to redistribute to lists, requires prior specific permission and/or a fee.

MobiSys'13, June 25-28, 2013, Taipei, Taiwan

Copyright 2013 ACM 978-1-4503-1672-9/13/06 ...\$15.00. phone for incoming text messages while in driving mode as discussed in $[26,36]$.

Such approaches depend on the phone being able to sense when the user is driving, since experience with a phone's silent mode and instant message status has shown that users are not very reliable at setting the status manually. Several known approaches exist for detecting whether a phone user is in a vehicle. More difficult, however, is determining whether a user is actually driving or is simply a passenger in the vehicle. In our prior work, we addressed this problem by exploiting built-in Bluetooth handsfree systems in vehicles for audio localization of the phone. While it is expected that the fraction of Bluetooth handsfree equipped vehicle will rise significantly over the coming years, there is also considerable interest in techniques that are less dependent on such infrastructure, so that they can be more easily retrofitted into existing vehicles without Bluetooth.

In this paper, we explore a low-infrastructure approach that senses acceleration due to vehicle dynamics to decide a phone's position. As in prior work, we seek to determine the in-vehicle location of the phone and use that as a heuristic to determine whether the phone is used by the driver or passenger. It uses a fundamentally different sensing approach, however, to determine this location. The key insight is that the centripetal acceleration varies depending on the position in the car. By comparing the measured acceleration from the phone with the acceleration measured at a reference point inside the car, the phone can decide whether it is located left or right of the reference (i.e. on the driver or passenger side). This technique can operate in conjunction with our bump sensing technique for determining front or rear location [37]. We refer to this approach as low-infrastructure since it can more easily be retrofitted in existing cars by plugging in a small OBD-II or cigarette light adapter. It does not require having a handsfree bluetooth kit wired into the existing car audio system.

While the idea of utilizing centripetal acceleration differentials between the target phone and the reference point seems simple, many challenges arise in practice. First, the embedded sensors are noisy and affected by unpredictable driving environments. A second challenge is minimizing the additional infrastructure needed beyond the phone. Third, the sensor readings in smartphones are pose dependent, thus cannot be directly used to represent the vehicle's dynamics. To address these issues, we propose a centripetal acceleration based driver phone use sensing algorithm that mitigates the noise of the sensor readings and unpredictable geometries, 
such as different size of turns, driving speed, and driving styles. Extensive experimental results in both parking lots and roads confirm the effectiveness and efficiency of our proposed algorithm which requires no built-in infrastructure. Specifically, we make the following contributions:

- Proposing a method for distinguishing driver and passenger phone use that is less dependent on built-in vehicle infrastructure than the audio-ranging approach. It determines the in-vehicle position of a phone by using its sensors to monitor position dependent differences in acceleration forces and comparing them with a vehicle reference reading.

- Describing and evaluating multiple possible designs for providing a vehicle reference reading, including a cigarette lighter adapter with accelerometer sensor, an OBD-II port adapter that provides vehicle speed reference readings to the phone over Bluetooth, and opportunistically using other phones as a reference.

- Designing algorithms that can use these various reference inputs and can compensate for bias in the reference measurements by taking into account data from both left and right turns of a vehicle.

- Demonstrating through extensive experiments in a parking lot and through real-world driving in two cities with different phone models and vehicles that it is feasible to determine phone position with high accuracy after the vehicle has passed through a few curves.

The experimental results show that by relying only on the plug-in adapters, our proposed algorithm can achieve high detection rates and low false positive rate in both parking lot and real driving environments. By making use of a few turns, a more reliable result can be obtained. Our results show that position can often be determined after the vehicle drives out of a parking lot or before it reaches a main road. We therefore believe that the detection latency is acceptable. Our prototype implementation also shows that current Android smartphones have adequate computational capabilities to perform the signal processing needed in a standard programming environment.

The rest of the paper is organized as follows. In Section 2, we place our work in the broader context of reducing driver distraction and using sensors on smartphones to facilitate driving safety and vehicle monitoring applications. We provide the system overview and core detection algorithm in Section 3. We then present our system implementation by leveraging different infrastructures in Section 4. In Section 5, we perform extensive evaluation of our system in real-road driving environments involving two types of phones and two vehicles in two cities. We discuss about a possible infrastructure-free approach of determining driver phone use by adding a phone's GPS in Section 6. Finally, we conclude our work in Section 7.

\section{RELATED WORK}

There has been active research work in detecting dangerous behavior while operating an automobile [18,20,24], especially for the driver distraction problem caused by hand-held devices. Some recent work dedicated to mitigate driver phone distraction includes Quiet Calls [31], Blind Sight [25], Negotiator [35], and Lindqvist's systems [26]. Furthermore, some apps are developed to block incoming or outgoing calls and texts for the phones inside a moving vehicle $[5,12,14]$. Apps such as [9] require special devices

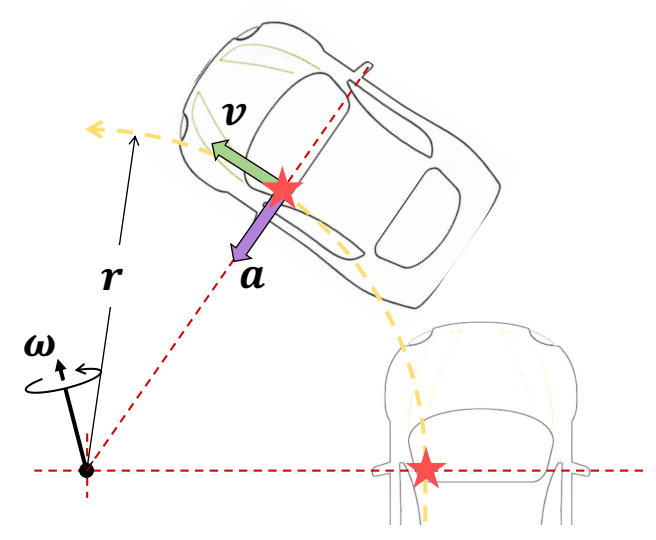

Figure 1: Illustration of centripetal acceleration, tangential speed, angular speed, and the radius of the circular movement.

installed inside the vehicle to enable blocking cellular communications of a specific phone based on the readings from the vehicle's speedometer, or even rely on a radio jammer [7]. These studies either require prior knowledge of the phone use by the driver (e.g., user activates the system indicating himself as the driver) or blindly block calls/text of all the cellphones inside the vehicle. These solutions, however, cannot automatically distinguish a driver's cell phone from a passenger's.

Since diverse sensors have been integrated in smartphones, they are endowed with powerful capabilities that can be used to sense vehicle dynamics and facilitate a broad array of applications related to driving safety and road monitoring. The moving vehicle can be detected based on the embedded smartphone sensors and the cellular signal, for example, accelerations and cellular signal strength $[15,23,30,33]$. Other studies use smartphone embedded sensors to alert dangerous driving, monitor road conditions, and detect traffic accidents [17,24,29,34]. Dai et.al. [17] propose a system to detect and alert dangerous vehicle maneuvers by utilizing the accelerometer and the orientation sensor. WreckWatch [34] detects traffic accidents using the accelerometer and microphone. Johnson et.al. [24] present a system using Dynamic Time Warping (DTW) and smartphone based sensor-fusion to detect and recognize vehicular motions. Furthermore, smartphone sensors are also used to monitor the road conditions [19,28, 29], e.g., the accelerometer in a smartphone is able to detect a large acceleration perpendicular to the road surface when the vehicle passes bumps or potholes on the road. These studies confirm the feasibility of utilizing sensors on smartphones to sense the vehicle dynamics, which may be further used to automatically determine the driver phone use.

Towards the most related work in detecting driver phone use, Chu et.al. [16] present a driver detection system (DDS) by utilizing multiple sensors (including accelerometer, gyroscope, and microphone) in smartphones to capture the features of driver's movement. However, this approach is sensitive to the behavior of each individual, and highly depends on the position where drivers carry the phone, which is less practical. Yang et.al. [36,37] introduce an acoustic relative-ranging system that classifies on which car seat a phone is being used leveraging the car's audio infrastructure. This approach relies on the vehicle's audio system. Different from the above work, in this project we explore a low-infrastructure approach that senses acceleration forces from vehicle dynamics to determine the phone's position inside the car.

\section{SYSTEM DESIGN}

To build a low-infrastructure solution leveraging embedded sensors on smartphones, we devise an approach that senses position- 


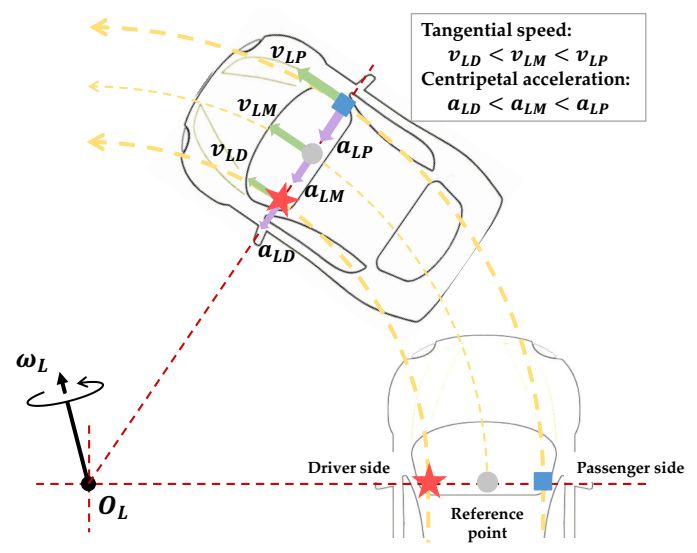

Figure 2: Illustration of different centripetal accelerations at different in-vehicle positions.

dependent acceleration forces when a vehicle turns. In this section, we provide background information, system challenges, overview of our system design, and a driver phone use sensing algorithm.

\subsection{Background}

When a vehicle makes a turn, it experiences a centripetal force, which has its direction orthogonal to the direction of movement of the vehicle and toward the center of the turn. This centripetal force generates a centripetal acceleration $a$ also pointing toward the center of the curve. Assuming a turn following a perfect circle, the centripetal acceleration $(a)$ can be obtained by using the angular speed $(\omega)$, the tangential velocity $(v)$ and the radius $(r)$ of the turn [32]:

$$
a=\omega v=\omega^{2} r=\frac{v^{2}}{r} .
$$

The relationship of these parameters are illustrated in Figure 1. Phones located on passenger- and driver-side positions inside the vehicle will have the same angular speed but follow circles of different radii. Based on Equation (1), it can be seen that different radii at constant angular speed will lead to differences in centripetal acceleration on these positions.

Inspired by this simple yet useful physics observation, we seek to measure such centripetal acceleration differences with smartphone sensors to design a low-infrastructure solution for determining driver phone use.

\subsection{Challenges and Goals}

Building such a system involves a number of challenges in both design and implementation:

Robustness to Real-Road Driving Environments. The centripetal acceleration is affected by a number of factors including the different size of turns, driving speed, and driving style. Furthermore, vibrations from the vehicle (e.g., a running engine) and environment (e.g., wind) all contribute to noisy sensor readings. Thus, the algorithm to obtain the centripetal acceleration has to be robust to deal with real road driving environments.

Achieving Single Phone Sensing. The approach should work even when only a single phone is present in the vehicle, since it is not always clear that this phone belongs to the driver.

Determining the Pose of the Phone. The measured sensor readings from smartphones can not be directly applied to produce vehicle dynamics (e.g., centripetal acceleration) without knowing the pose of the phone inside the vehicle. An effective re-orientation

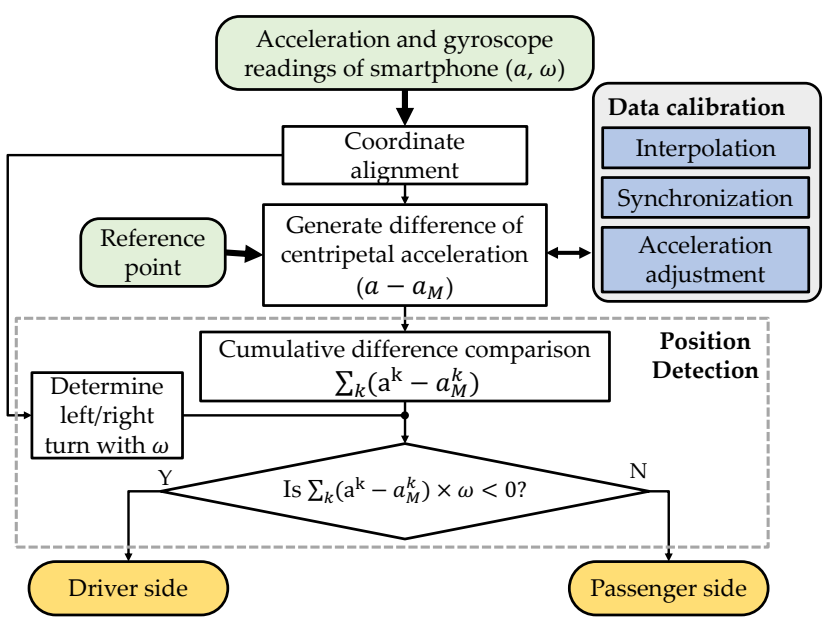

Figure 3: Overview of system flow.

mechanism is needed to align the phone's pose with the vehicle's coordinate system.

Computational Feasibility on Smartphones. The driver phone sensing process should complete in a short time on standard smartphone platforms.

\subsection{System Overview}

The basic idea of our system is to examine the centripetal acceleration (sensed by the smartphones during a turn) at different positions inside the vehicle. Figure 2 shows when a vehicle making a left turn, the driver side has smaller centripetal acceleration $\left(a_{L D}\right)$ than that at the vehicle's center $\left(a_{L M}\right)$, which in turn has smaller centripetal acceleration than that at the passenger side $\left(a_{L P}\right)$. In general, compared to the center of the vehicle the driver phone always has a smaller radius (and thus experiences smaller centripetal acceleration) when the vehicle makes a left turn and a larger radius (corresponding to larger centripetal acceleration) when the vehicle makes a right turn. Therefore, if the phone's centripetal acceleration is smaller than that at the center in a left turn, or larger in the case of a right turn, then the phone is on the left side of the vehicle. Our system can thus utilize the difference of the centripetal acceleration experienced at different positions within the vehicle to distinguish the driver phone use from passengers'.

A vehicle usually undergoes multiple turns at the beginning of a trip (e.g., pulling out of a parking lot or driving in local streets before getting onto main roads). The difference of the phone's centripetal acceleration obtained by comparing left and right turns can help to determine whether the phone is at the driver side or passenger side. However, this approach requires turns made by the vehicle have the same radii, which is not practical in real-road driving environments. Our system seeks to find a solution that can exploit the difference of centripetal acceleration from the same turn to sense the driver phone use. The advantage of this solution is that our system can work under real-world driving scenarios with various turn sizes and driving speeds.

In particular, our system can obtain the difference of the centripetal acceleration within a turn by employing a reference centripetal acceleration, such as that at the vehicle's center or from a second phone. There are three ways of utilizing a reference centripetal acceleration that we implement in our system: 1) A lowcost cigarette lighter adapter containing an accelerometer acting as a reference point. Our system can directly compare the centripetal acceleration of the phone in the vehicle to that obtained from the 
cigarette lighter adapter to determine the driver phone use. 2) The speed of the vehicle obtained from the OBD-II port adapter can be used to calculate the centripetal acceleration at the vehicle's center, which is compared to the centripetal acceleration of the smartphone to detect the driver phone use. The OBD-II interface has been made mandatory for all vehicles sold in the United States since 1996. 3) When there are multiple occupants in the car, a passenger phone in the same vehicle can be employed. Our system can compare the centripetal acceleration of the passenger phone and that of the driver phone. This approach, however, relies on the presence of (and communicating to) a second phone in the vehicle. In this work, we focus on solutions (i.e., solutions 1 and 2) that a single phone can perform self-determination of the driver phone use through sensing. The plug-in adapters could share the reference readings with the phone over Bluetooth.

Realizing our approach requires three sub-tasks: Coordinate Alignment, Data Calibration, and Position Detection. The flow of our system is illustrated in Figure 3. When the target phone detects the Bluetooth connection (e.g. from the cigarette lighter adapter when the driver enters the car), it starts collecting readings from its accelerometer and gyroscope, which are used to derive the phone's acceleration and angular speed. Our system performs Coordinate Alignment so that the centripetal acceleration and angular speed derived from the phone sensors are aligned with the vehicle's. The coordinate alignment is only performed when our system starts or when the system detects the gyroscope readings crossing certain thresholds, which is caused by the change of phone's position. Once the vehicle is detected to start making a turn, the target phone collects the information from the reference point (e.g., acceleration from cigarette lighter adapter or speed from the OBD-II port adapter). The phone further conducts calibration on the data collected by itself as well as the data reported by the reference point. Our data calibration process includes three steps: Data Interpolation, Trace Synchronization, and Acceleration Adjustment, which aims to synchronize the traces from different sources and reduce the hardware bias caused by different phone models. Finally, Position Detection determines the phone's position in car leveraging the cumulative difference of centripetal acceleration (e.g., $k$ samples around the maximum angular speed) and combining the turn direction determined from the sign of the angular speed. We next describe how to sense vehicle dynamics using smartphones and present the core component, Detection Algorithm, in our system. We leave the detailed presentation of Coordinate Alignment and Data Calibration to the next section (Section 4).

\subsection{Sensing Vehicle Dynamics}

Phone and Vehicle Alignment. We utilize the 3-axis accelerometer and 3-axis gyroscope embedded in the smartphone to obtain the centripetal acceleration while the vehicle makes a turn. There are two coordinate systems, one for the smartphone $\left(\left\{X_{p}, Y_{p}, Z_{p}\right\}\right)$ and the other for the vehicle $\left(\left\{X_{c}, Y_{c}, Z_{c}\right\}\right)$, as illustrated in Figure 4. To simplify the description of our approach, we assume the smartphone's coordinate system is already aligned with the vehicle's (i.e., aligned). We will describe how to align the phone's coordinate system in Section 4.2.

Deriving Centripetal Acceleration via Accelerometers. As illustrated in Figure $4, X_{c}$ points to the passenger side of the vehicle (i.e., opposite side of the driver). The $X$-axis acceleration reading on the phone reflects the centripetal acceleration (i.e., $a$ ) when the vehicle makes a turn. As illustrated in Figure 5, the $X$-axis reading is zero when the vehicle is driving along a straight line and reaches its positive or negative peak when the vehicle goes into the middle of a turn. The sign of the acceleration on the $X$-axis is determined

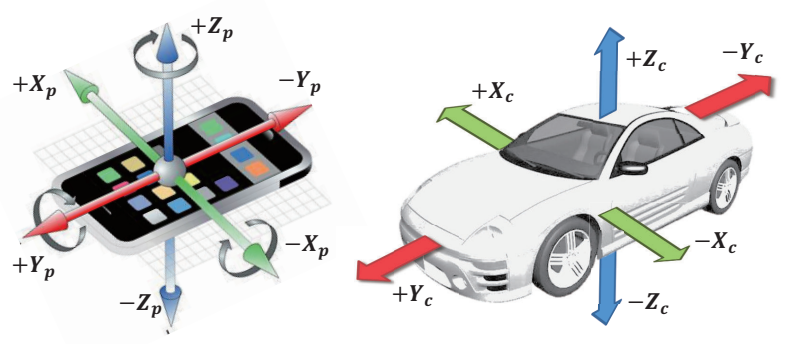

Figure 4: Coordinate systems of a smartphone and a vehicle.

by the turn direction due to that the centripetal acceleration is always pointing to the center of a turn. Thus, the $X$-axis acceleration is negative when the vehicle is making a left turn, and vice versa. Additionally, the $Y_{c}$ points to the head of the vehicle. Thus, the $Y$-axis acceleration reading of the phone indicates the acceleration of the tangential speed (i.e., $v$ ) of the vehicle in a turn.

Determining Turn Directions using the Gyroscope. To compare the centripetal acceleration at different positions inside the vehicle, we need to determine the turn direction, i.e., whether the vehicle is making a right turn or a left turn. The $Z$-axis gyroscope reading on the phone can be utilized to represent the vehicle angular speed of the turn. Figure 5 illustrates the rotation rate on $Z$-axis of a gyroscope on the phone during a left and right turn respectively. A counter clockwise rotation around $Z$-axis generates positive reading, which indicates the vehicle is making a left turn; otherwise, the gyroscope generates negative reading, indicating the vehicle is making a right turn. We note that the power consumption is only $1.5 \mathrm{~mW}$ [8] for a accelerometer sensor and $10 \mathrm{~mW}[8,21]$ for a gyroscope sensor. Whereas the power consumption of a smartphone is significantly higher (for example, the average power of HTC EVO is about $450 \mathrm{~mW}$ [27]).

\subsection{Algorithm for Sensing Driver Phone Use}

It is essential to understand what are the important factors affecting the difference of the centripetal acceleration between two different positions inside the vehicle. We have the following lemma to capture such factors:

LEMMA 1. The difference of centripetal acceleration between two in-vehicle positions is determined by the angular speed and relative distance between these two positions.

PROOF. Assume there are two positions in the vehicle, one is the target position which is unknown, and the other is a known reference position, e.g., the center of the vehicle. When the vehicle is making a left turn, assume the radius of the target phone is $r_{L}$, and the radius of the reference position is thus $r_{L M}=r_{L}+\Delta r$, where $\Delta r$ is the relative distance between the target position and the reference position. The difference of centripetal acceleration between these two positions can then be represented as

$$
\Delta a_{L}=a_{L}-a_{L M}=\omega_{L}^{2} r_{L}-\omega_{L}^{2}\left(r_{L}+\Delta r\right)=-\omega_{L}^{2} \Delta r .
$$

Similarly, when the vehicle is making a right turn, the difference of centripetal acceleration between the target phone and the reference position is $\Delta a_{R}=\omega_{R}^{2} \Delta r$. Based on the equations above, it is clear that the difference of the centripetal acceleration between two positions inside the vehicle is determined by the angular speed of the vehicle and the distance between these two positions.

The above analysis shows that the difference of centripetal acceleration only depends on the relative distance between two positions inside the vehicle and angular speed during the turn. Thus, 

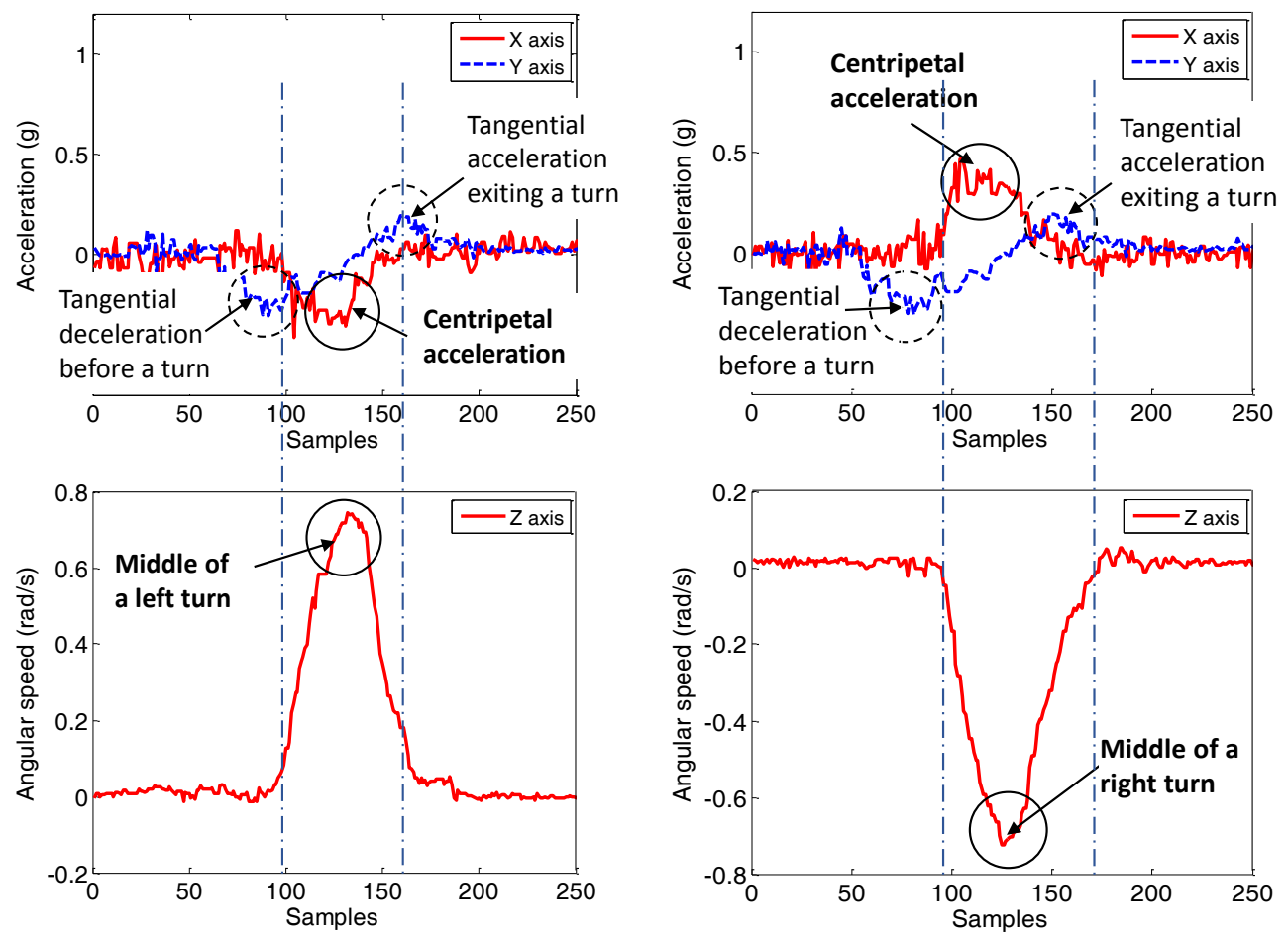

Left turns

Right turns

Figure 5: Accelerometer and Gyroscope readings when a smartphone is aligned with the vehicle who undergoes a left and a right turn respectively.

our approach of using the difference of centripetal acceleration is scalable to handle any turns with different radii. The larger the angular speed is, the more powerful the discrimination becomes in the centripetal acceleration when sensing driver phone use. Moreover, when undergoing left turns, the centripetal acceleration of the driver phone is smaller than that at the reference point (such as the cigarette lighter adapter and OBD-II port adapter), whereas it is larger than that of the reference point when going through right turns. Therefore, given the difference of the centripetal acceleration and the turning direction, our system is able to determine whether the phone is a driver phone or passenger one. Specifically, our algorithm determines the driver phone use within a single turn using the following hypothesis test:

$$
\left\{\begin{array}{l}
\left(a-a_{M}\right) \omega>0, \mathcal{H}_{0}: \text { passenger phone } \\
\left(a-a_{M}\right) \omega<0, \mathcal{H}_{1}: \text { driver phone, }
\end{array}\right.
$$

where $a$ is the centripetal acceleration of the smartphone measured from its $X$-axis accelerometer, $a_{M}$ is the centripetal acceleration of the reference position, and $\omega$ denotes the angular speed measured from smartphone's $Z$-axis gyroscope sensor. The sign of $\omega$ reflects the turn direction, e.g., $\omega$ is positive when the vehicle is making a left turn.

Cumulative Difference Comparison. Finally, the differences of centripetal acceleration within the turning period are accumulated in our algorithm so that to improve the detection robustness. Particularly, our algorithm utilizes 21 samples of acceleration readings at the time when the angular speed reaches its maximum value. The cumulative difference of centripetal acceleration is then combined together with the turning direction to decide whether the target phone is on the driver side or the passenger side.

Feasibility Study. Figure 6 depicts the difference of centripetal acceleration between driver's phone and passenger's when our vehicle went through 57 left turns and 60 right turns respectively. The results are categorized in three ways: angular speed, car speed and turn radius. It is encouraging that there is an obvious trend that increasing angular speed results in a larger value of difference of centripetal acceleration (as observed in Figure 6 (a)). Whereas the difference does not change much when increasing the vehicular driving speed and turn radius as shown in Figure 6 (b) and (c).

Utilizing Multiple Turns. Our algorithm can further improve the detection performance by combining multiple single turn results (e.g., $N$ turns) through a simple majority voting process:

$$
\left\{\begin{array}{l}
\sum_{i=1}^{N} \frac{\left(a^{i}-a_{M}^{i}\right) \omega^{i}}{\left|\left(a^{i}-a_{M}^{i}\right) \omega^{i}\right|}>0, \mathcal{H}_{0}: \text { passenger phone } \\
\sum_{i=1}^{N} \frac{\left(a^{i}-a_{M}^{i}\right) \omega^{i}}{\left|\left(a^{i}-a_{M}^{i}\right) \omega^{i}\right|}<0, \mathcal{H}_{1}: \text { driver phone }
\end{array}\right.
$$

where $a^{i}, a_{M}^{i}$, and $\omega^{i}$ are the smartphone's centripetal acceleration, reference centripetal acceleration, and smartphone's angular speed in $i^{t h}$ turn.

\subsection{Detection Using Mixed Turns}

The accuracy of the reference point affects the performance of our sensing algorithm. We find that observations from the reference point can be biased. For example, the vehicle speed provided by OBD-II is an overestimation possibly due to worn tires. Such a bias affects the algorithm accuracy when using the difference of centripetal acceleration within the same turn. Since a vehicle usually undergoes multiple turns during a trip, we exploit the centripetal acceleration obtained from mixed turns, i.e., comparing the normalized centripetal acceleration of the phone under a left turn to that of a right turn. The normalized centripetal acceleration 


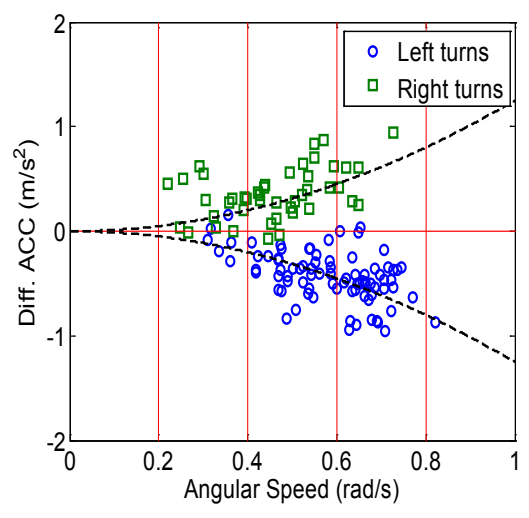

(a) Categorized with angular speed

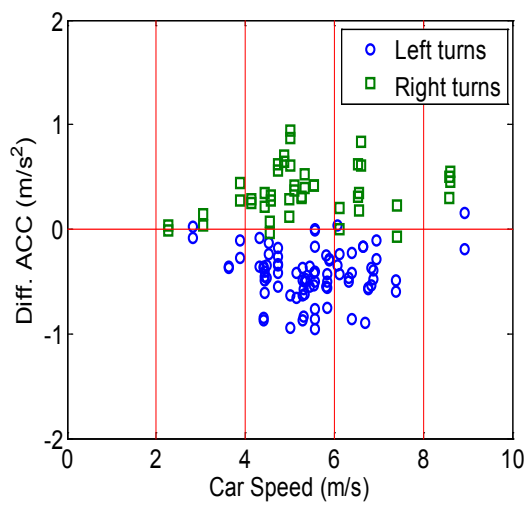

(b) Categorized with car speed (obtained from OBD-II)

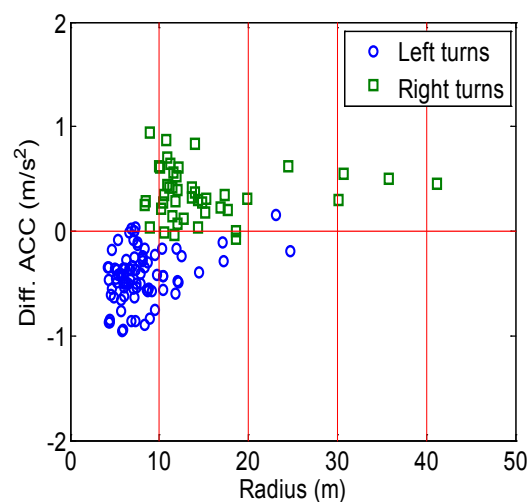

(c) Categorized with turn radius

Figure 6: Difference of centripetal acceleration between driver's phone and passenger's categorized in three ways (angular speed, car speed, and turn radius) when the vehicle undergoes 57 left turns and 60 right turns in the parking lot.

is defined as the ratio of the measured centripetal acceleration of the phone to the centripetal acceleration derived from the reference point. Using normalized centripetal acceleration enables our algorithm to work with mixed turns with different turn sizes and driving speeds encountered under real-road driving environments. The system can automatically launch this detection once a left turn and a right turn are identified based on gyroscope readings, irrespective of the sequence of these turns.

Impact of Bias. The reference centripetal acceleration $a_{L M}^{\prime}$ (for example under the left turn) can be expressed as:

$$
a_{L M}^{\prime}=a_{L M} \beta
$$

where $a_{L M}$ is unbiased centripetal acceleration of the reference point and $\beta$ is the bias. When the OBD-II port adapter is used as the reference point, $\beta$ comes from the biased estimate of the vehicle speed. Then the difference in centripetal acceleration becomes:

$$
\Delta a_{L}=a_{L}-a_{L M}^{\prime}=(1-\beta) a_{L}-\beta \omega_{L}^{2} \Delta r .
$$

When there is no bias (i.e., $\beta=1$ ), the above expression becomes Equation (2). However, the existence of bias $(\beta \neq 1)$ can arbitrarily change the sign of the difference in centripetal acceleration, making the detection result inaccurate.

Working with Mixed Turns. Our algorithm compares the normalized centripetal acceleration of the phone under a left turn to that of a right turn to eliminate the impact of bias coming from the reference point. We denote the normalized centripetal acceleration of the phone under a left and right turn as $\hat{a}_{L}=\frac{a_{L}}{a_{L M}^{\prime}}$ and $\hat{a}_{R}=\frac{a_{R}}{a_{R M}^{\prime}}$, respectively. The difference of the normalized centripetal acceleration under the left and right turn can then be expressed as:

$$
\begin{aligned}
\Delta \hat{a}_{r} & =\hat{a}_{L}-\hat{a}_{R}=\frac{a_{L}}{a_{L M}^{\prime}}-\frac{a_{R}}{a_{R M}^{\prime}}=\frac{a_{L}}{a_{L M} \beta}-\frac{a_{R}}{a_{R M} \beta} \\
& =\frac{1}{\beta}\left(\frac{a_{L}}{a_{L M}}-\frac{a_{R}}{a_{R M}}\right) .
\end{aligned}
$$

If the phone is at the driver side, $a_{L M}$ is always larger than $a_{L}$ (i.e., $\frac{a_{L}}{a_{L M}}<1$ ), whereas $a_{R M}$ is always smaller than $a_{R}$ (i.e., $\frac{a_{R}}{a_{R M}}>1$ ). Thus, we always have $\Delta \hat{a}_{r}<0$. Similarly, if the phone is at the passenger side, we always have $\Delta \hat{a}_{r}>0$. Thus, the sign of $\Delta \hat{a}_{r}$ becomes independent of the bias, turn size and driving speed. Our driver phone sensing with mixed turns can be further formulated as the following hypothesis test:

$$
\left\{\begin{array}{l}
\hat{a}_{L}-\hat{a}_{R}>0, \mathcal{H}_{0}: \text { passenger phone } \\
\hat{a}_{L}-\hat{a}_{R}<0, \mathcal{H}_{1}: \text { driver phone. }
\end{array}\right.
$$

We envision that our system can intelligently perform driver phone detection based on the availability of turns. This means that when a single turn is available, our system applies the algorithm involves the single turn. When multiple/mixed turns are available, our system performs more accurate driver phone detection using the accumulative multiple/mixed turns.

\section{SYSTEM IMPLEMENTATION}

In this section, we present the Data Calibration and Coordinate Alignment sub-tasks in our system. We then describe two system approaches, one using the cigarette lighter adapter with an accelerometer sensor and the other using the OBD-II port adapter as the reference points.

\subsection{Data Calibration for Enhanced Reliability}

In real-road driving environments, many factors (such as running engines and wind) affect the readings from the accelerometers and gyroscopes on smartphones. The sensor readings obtained can be noisy and unreliable. To address this issue, we develop several steps in our system to perform data calibration for robust detection. Our data calibration sub-task has the following capability: filter noise from sensor readings, ensure the synchronization between sensor readings from different sources, and reduce bias caused by hardware difference in smartphones.

\subsubsection{Data Interpolation}

To reduce the noise in readings obtained from the accelerometers, we apply a moving average filter to the sensor readings. However, we observe that although a fixed sampling rate is used, the real sampling interval has a small variation. Therefore, before applying the moving average filter, we interpolate to estimate the samples at evenly spaced time series points, i.e. $\left[t_{0}, t_{0}+\delta, t_{0}+2 \delta, \ldots\right]$, where $\delta$ is the interpolation step and $t_{0}$ is the starting time stamps for the 


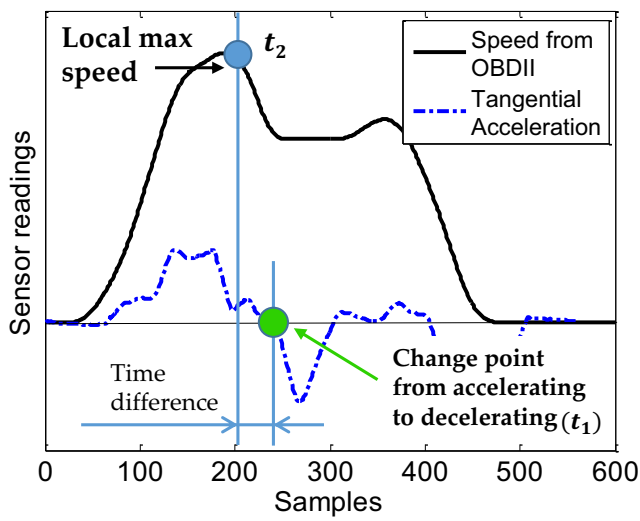

(a) Before synchronization

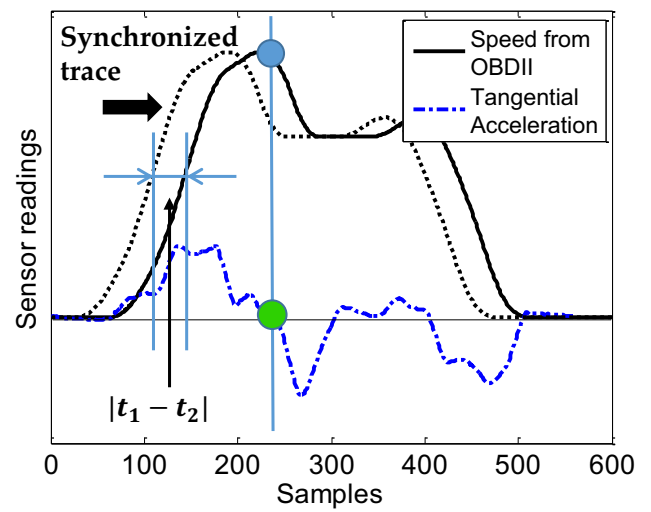

(b) After synchronization

Figure 7: Illustration of trace synchronization mechanism via tangential acceleration.

readings. Similarly, we also apply interpolation to readings from the gyroscope to obtain a uniform time interval between consecutive samples for comparison. In our experiments, we observe that a small time window of 5 samples for the moving average filter and a $\delta$ of $0.05 \mathrm{~s}$ for the interpolation step are good enough to produce reliable sensing results.

\subsubsection{Trace Synchronization}

This procedure is used to synchronize the sensor readings from the phone and the readings at the reference point (e.g., the cigarette lighter adapter or OBD-II port adapter) since these readings come from two sources with different clocks. In our approach, two types of reference data are involved, one is the centripetal acceleration of the vehicle (reference acceleration from the cigarette lighter adapter), and the other is the speed of the vehicle (reference speed from OBD-II port adapter). To synchronize the phone's centripetal acceleration readings with the ones from the reference acceleration we calculate the cross correlation between these two sequence of readings in time series. When the cross correlation reaches the maximum, these two sequence of readings are synchronized because both sequences reflect vehicle's movement.

However, when the speed obtained from the OBD-II port adapter is used as the reference point, synchronization becomes more challenging. We develop a synchronization mechanism utilizing vehicle's acceleration, leveraging the change point in the tangential acceleration during normal driving, to synchronize the trace of reference speed from OBD-II with the acceleration reading trace from
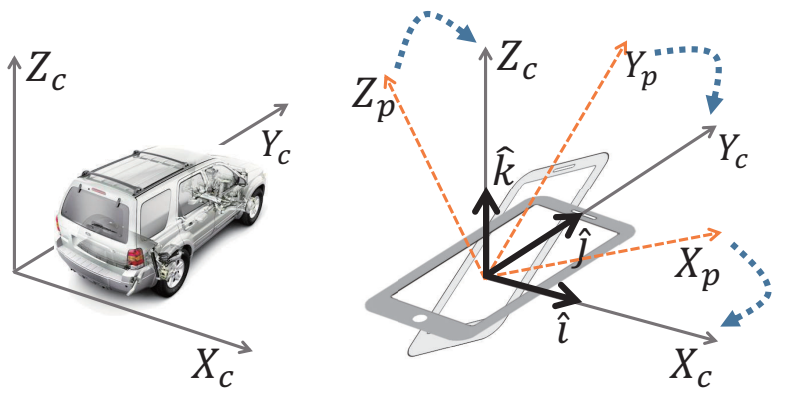

Figure 8: Illustration of how the phone's coordinate system is aligned to the vehicle's coordinate system.

smartphone in time series. The rationale behind this mechanism is that the time point that the vehicle changes from acceleration to deceleration during normal driving is the point that the vehicle reaches its maximum speed. Figure 7 illustrates how the tangential acceleration value change facilitates the synchronization with the reference speed trace. The time $\left(t_{2}\right)$ that the reference speed from OBD-II reaches its local maximum should match the time $\left(t_{1}\right)$ that the vehicle's tangential acceleration (i.e. the acceleration on the $Y$ axis) changes from positive to negative. Thus, for the reference speed trace (from OBD-II), we can perform synchronization by subtracting the time difference $\left(t_{2}-t_{1}\right)$ from all its time stamps.

\subsubsection{Acceleration Adjustment}

Acceleration adjustment is used to reduce the bias caused by hardware differences in smartphones through adjusting the centripetal acceleration of the phone. Because the centripetal acceleration only exists during a turn, the readings on the $X$-axis accelerometer of the phone should be zero when the vehicle is moving along a straight line. Nevertheless, the acceleration on the $X$ axis may have a constant value different from zero due to different hardware characteristics in different phone models. To reduce such a bias, our system performs the following adjustment: 1) use the phone's gyroscope to determine the time period that the vehicle is driving along a straight line, i.e., the time period with no rotation rate on the $Z$-axis gyroscope; 2 ) calculate the mean value of the $X$-axis acceleration during this time period; and (3) subtract the calculated mean value from all the $X$-axis acceleration readings to remove the constant bias.

\subsection{Coordinate Alignment}

Our system cannot derive meaningful vehicle dynamics from sensor readings on the smartphone unless the phone's coordinate system is aligned with the vehicle's. The Coordinate Alignment sub-task aligns the phone's coordinate system with the vehicle's by utilizing the accelerometers and gyroscopes on smartphones. As illustrated in Figure 8, the phone's coordinate system $\left(\left\{X_{p}, Y_{p}, Z_{p}\right\}\right)$ is determined by the pose of the phone inside the vehicle. Our coordinate alignment aims to find a rotation matrix $R$ to rotate the phone's coordinate system to match with the vehicle's $\left(\left\{X_{c}, Y_{c}, Z_{c}\right\}\right)$. We define three unit coordinate vectors under the vehicle's coordinate system as $\hat{i}, \hat{j}$ and $\hat{k}$ for $X_{c}, Y_{c}$ and $Z_{c}$ axis respectively (i.e., $\hat{i}=[1,0,0]^{T}$ in vehicle's coordinate system). We denote the corresponding coordinates of these three unit vectors in the phone's coordinate system as:

$$
\hat{q}=\left[x_{q}, y_{q}, z_{q}\right]^{T},
$$




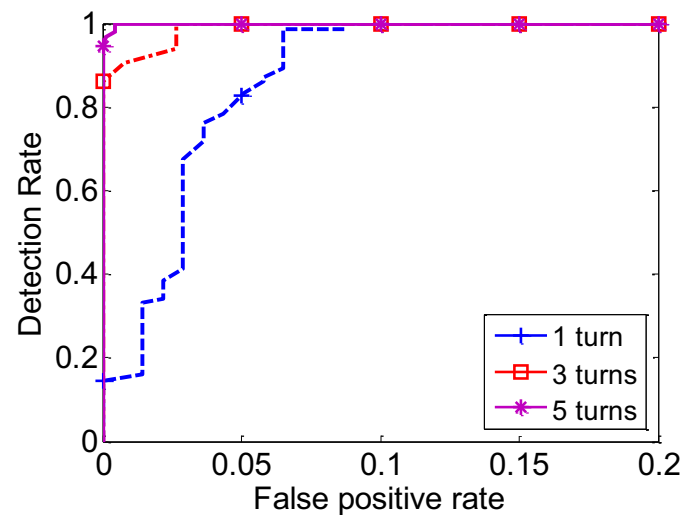

Figure 9: ROC curve when using single or multiple turns and the acceleration of adapter phone as the reference acceleration in parking lot.

where $q \in i, j, k$, and the rotation matrix is given by [22]:

$$
R=\left\{\begin{array}{lll}
x_{i} & x_{j} & x_{k} \\
y_{i} & y_{j} & y_{k} \\
z_{i} & z_{j} & z_{k}
\end{array}\right\}
$$

Our coordinate alignment sub-task utilizing smartphone's accelerometer and gyroscope readings to obtain each element in the rotation matrix $R$ consists of three steps:

Deriving $\hat{k}$. We can apply a low pass filter (e.g., exponential smoothing) on the three axes accelerometer readings on the phone to obtain the constant components from these three accelerations and derive the gravity acceleration [1], which is then be normalized to generate the unit vector $\hat{k}=\left[x_{k}, y_{k}, z_{k}\right]^{T}$.

Deriving $\hat{j}$. To obtain $\hat{j}$, we utilize the fact that the three-axes accelerometer readings of the phone are caused by vehicle's acceleration or deceleration when driving straight. For example, we can obtain $\hat{j}=\left[x_{j}, y_{j}, z_{j}\right]^{T}$ through extracting the accelerometer readings when the vehicle decelerates (e.g., the vehicle usually decelerates before making turns or encountering traffic lights and stop sign). The gyroscope is used to determine whether the vehicle is driving straight (i.e., with zero rotation rate). We note the gravity component needs to be excluded because it distributes on all three axes of the phone when the phone's coordinate system is not aligned with the vehicle's.

Obtaining $\hat{i}$. Since the coordinate system follows the right hand rule, we can determine the unit vector $\hat{i}=\hat{j} \times \hat{k}=\left[x_{i}, y_{i}, z_{i}\right]^{T}$.

After obtaining the rotation matrix $R$, given the sensor reading vector in the phone's coordinate system $\mathbf{s}$, we can obtain the rotated sensor reading vector $\mathbf{s}^{\prime}$ aligned with vehicle's coordinate system by applying a rotation matrix $R$ as: $\mathbf{s}^{\prime}=\mathbf{s} \times R$. We note that there are existing studies utilizing the sensors embedded in smartphones to calibrate the coordinate systems between the phone and the vehicle [29]. Different from the previous study, our coordinate alignment mechanism does not require working with the GPS on the phone, and thus is more accurate and energy efficient.

\subsection{Reference Using a Cigarette Lighter Ad- apter}

We next show how a low-cost cigarette lighter adapter containing an accelerometer can be employed as a reference point in our system. The location of the cigarette lighter charger is ideal for the reference point since it is located at the center of the front seats.

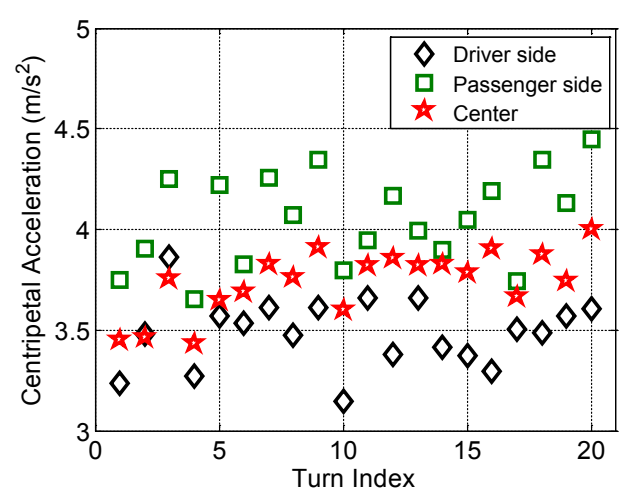

(a) 20 left turns

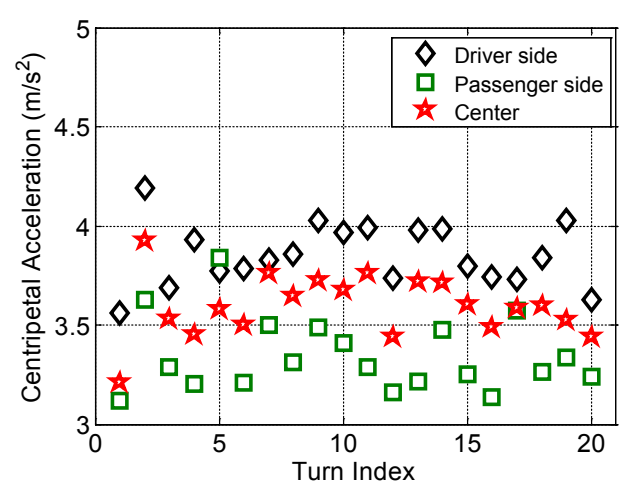

(b) 20 right turns

Figure 10: Centripetal acceleration at different positions inside the vehicle under 20 left and 20 right turns in parking lot.

Our system can thus distinguish driver phone use from passenger's by comparing the centripetal acceleration of the phone to that of the reference point. The centripetal acceleration of the reference point can be obtained from the cigarette lighter adapter's accelerometer. The measured centripetal acceleration from the cigarette lighter adapter is then transmitted to the target phone via Bluetooth for comparison.

Since at the beginning of a trip, a vehicle usually makes multiple turns to pull out of a parking lot or drive on local streets before getting onto main roads, we demonstrate the feasibility of the cigarette-lighter-adapter-based approach by driving a car in the parking lot of Babbio Center at Stevens Institute of Technology for over a one month time period. During our experiments, we utilize a smartphone (the adapter phone) and place it at the location of the cigarette lighter charger to simulate the cigarette lighter adapter containing an accelerometer. To distinguish the driver phone use from the passenger's, we place two iPhone $4 \mathrm{~s}$ at driver and passenger side respectively. We have 65 turns in total including both left and right turns at the parking lot. Each turn has about 90 degrees and lasts for about 20 seconds (which includes the time period when driving straight and turning). The radii of the turns are approximately 10 meters and the speed of the turns is around $10 \mathrm{mph}$. Note that without notice, the sensor readings we are referring to are after coordinate alignment.

Figure 9 presents the detection rate versus false positive rate when applying driver phone sensing algorithm within the same turn. The detection rate indicates how many cases of driver phone use are correctly detected, whereas the false positive rate shows 


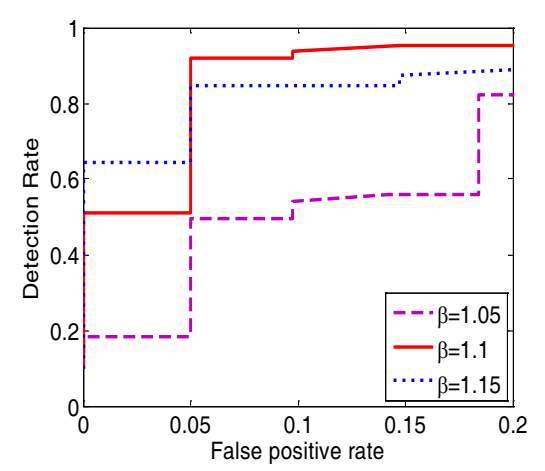

(a) 2 turns

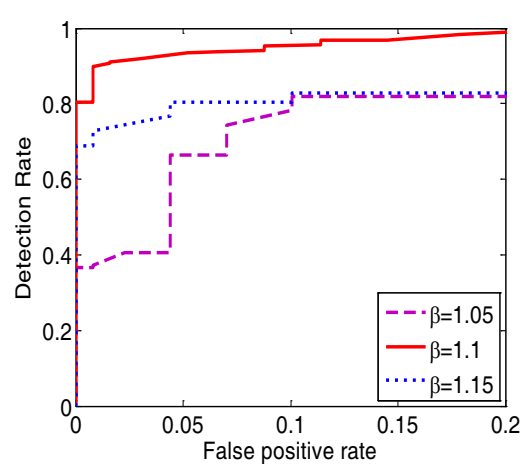

(b) 3 turns

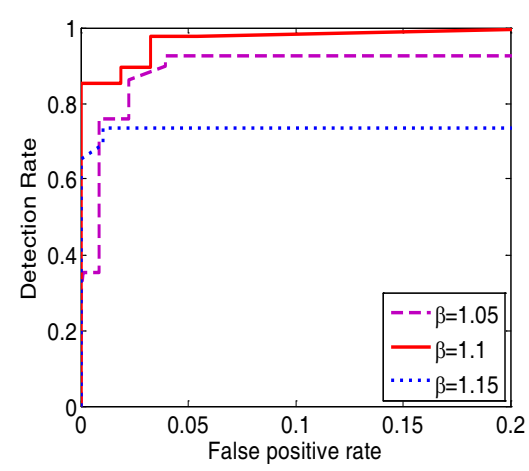

(c) 5 turns

Figure 11: ROC curve when using multiple turns and OBD-II speed in parking lot.

how many cases of passenger phone use are mistakenly classified as driver phone use. It is encouraging that our system can achieve above a $90 \%$ detection rate with about a $6 \%$ false positive rate using a single turn. Once the algorithm is applied when the vehicle undergoes multiple turns, the performance has a substantial improvement. For example, with 3 turns, the detection rate can be improved to $99.7 \%$ with much less false positive (3\%), whereas with 5 turns, our system can reach $100 \%$ detection rate with less than $1 \%$ false positive $(0.4 \%$ to be exact). These results confirms the feasibility of sensing vehicle dynamics to determine driver phone use.

\subsection{Reference Using an OBD-II Port Adapter}

The OBD-II interface has been made mandatory for all vehicles sold in the United States after 1996, and inexpensive OBD-II port adapters with Bluetooth connection are readily available in the market. We can forward the speed of the vehicle from the OBD-II port adapter to the smartphone via a Bluetooth connection. In our system implementation, we utilize a low cost OBD-II port adapter, which allows us to collect the vehicle's speed from the OBD-II port adapter via a USB connection, to use the speed of the vehicle as the reference point. The centripetal acceleration of the car's center (i.e., reference point) is the product of the OBD-II speed and the angular speed measured by the target phone. The driver phone use can be detected by comparing the phone's centripetal acceleration to that of the vehicle's center.

\subsubsection{OBD-II Speed}

We use the ElmScan 5 compact OBD-II scan toolkit (about 30 dollars) to obtain the OBD-II speed, which has a sampling rate of about 20 samples/s. The OBD-II speed represents the speed of the car's center since it is calculated based on the averaged distance traversed by four tires. Thus, the calculated centripetal acceleration based on the OBD-II speed and the angular speed is for the center of the vehicle. However, the OBD-II speed estimation is a conservative overestimation to allow for changes in tires' circumferences. We assume the reported speed is proportional to the true speed: $v_{M}^{\prime}=v_{M} \times \beta$, where $v_{M}^{\prime}$ and $v_{M}$ are the estimated and true speed respectively. We also assume that the value of $\beta$ is constant per vehicle, even though there is a slight variation in practice due to the change of tire pressure. We study the impact of the bias $(\beta)$ on the performance of our algorithm in our experiments.

\subsubsection{Evaluation}

We use the same experiment setup as we had in the parking lot in Section 4.3. Figure 10 shows the scatter plot on the centripetal accelerations from three sources: smartphone at driver seat, smart- phone at passenger seat, and the calculated one for the vehicle's center based on the OBD-II speed, under 20 left turns and 20 right turns respectively. Note that we did the OBD-II speed adjustment by setting the speed adjustment coefficient $\beta=1$.1. We observe that the centripetal acceleration calculated based on OBD-II speed is in between the centripetal acceleration of the driver phone and passenger phone. This indicates that employing the centripetal acceleration derived from the OBD-II speed is an effective reference point for determining driver phone use.

Figure 11 depicts the detection rate versus false positive rate by applying our algorithm when using multiple turns under different bias $\beta$. With a small bias $\beta=1.1$, we can achieve $91 \%$ detection rate with $5 \%$ false positive rate under two turns. By increasing the number of turns, the performance can be significantly improved. For example, with 3 and 5 turns, the detection rate is improved to $93 \%$ with $5 \%$ false positive rate and $97 \%$ detection rate with only $3 \%$ false positive rate, respectively. We find that the bias $\beta$ is critical to the detection performance when only data for few turns is available, but the sensitivity to $\beta$ decreases with the increasing number of turns, as shown in Figure 11. When data for multiple turns is available, our approach does not rely on a careful calibration of $\beta$, rather, a simple approximation is sufficient. Note that the bias can be learned offline. Alternatively, our algorithm based on mixed turns (Section 3.6) can eliminate the impact of the biased OBD-II speed. We present the results from using mixed turns tested from real-road driving environments in the next section.

\section{EVALUATION IN REAL-ROAD DRIVING ENVIRONMENTS}

In this section, we evaluate our proposed driver phone use sensing system in real road driving environments using two types of phones in two different cities.

\subsection{Experimental Setup}

\subsubsection{Phones and Vehicles}

We conduct our experiments with two types of phones: iphone4 and HTC 3D. Both phones have a Bluetooth radio, 3-axis accelerometer and gyroscope. The iphone4 is equipped with a $1 \mathrm{GHz}$ ARM Cortex-A8 CPU and 512M RAM running with iOS5.2, whereas the HTC 3D has a Qualcomm MSM8660 1.2GHz CPU and 1G RAM running with Android 2.4. Both the accelerometer and gyroscope sampling rate are 20 samples/s. There are two vehicles used in our experiments: a Honda Accord $(\operatorname{Car} A)$ and an Acura sedan $(\operatorname{Car} B)$. 


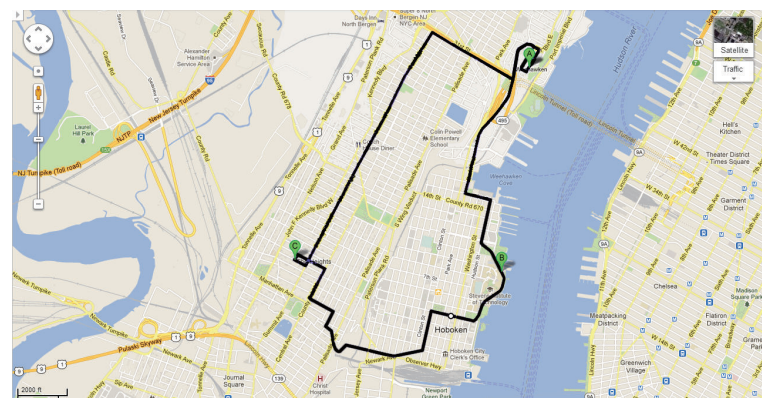

(a) Driving trajectory in Hoboken, NJ

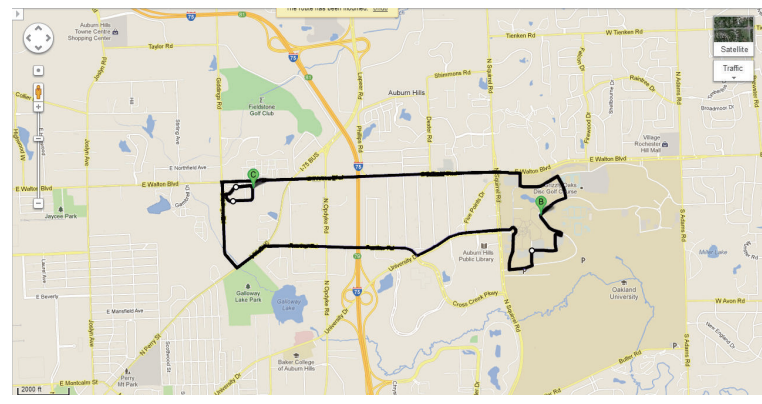

(b) Driving trajectory in Pontiac, MI

Figure 12: Daily commute routes used for real-road driving evaluation in Hoboken, NJ and Pontiac, MI.

\subsubsection{Real Road Driving Scenarios}

To evaluate our proposed system, we conduct experiments using the iphone4 for over one month when Car A is used as the daily commute vehicle in Hoboken, NJ. Hoboken has a typical urban setting. To test the generality of our system, we further experiment with the HTC 3D using Car B to commute to work in Pontiac, MI for over one week. Pontiac presents a suburban environment. The driving routes are depicted in Figure 12. The phones are placed in either the driver or passenger seat/door during the experiments. Each of the traces contains 10 to 20 -minute of driving. Table 1 summarizes the details on the traces collected in these two cities. In total, we have 292 left turns and 278 right turns in Hoboken, NJ, and 211 left turns and 219 right turns in Pontiac, MI.

\subsubsection{Prototype}

We implement our sensing driver phone use system using the Android platform. The prototype runs as an Android App and collects readings from the accelerometer and gyroscope in the smartphone. It then runs through the detection algorithm using either single or multiple turns to determine whether the phone is at the driver or passenger side. Our prototype also works with the OBD-II port adapter via Bluetooth. We also present the results using iPhone 4 by applying trace driven off-line analysis.

\subsubsection{Metrics}

\begin{tabular}{|c|c|c|c|c|}
\hline Location & Left turns & Right turns & Car & Phone \\
\hline \hline Hoboken,NJ & 292 & 278 & Car A & iphone4 \\
\hline Pontiac,MI & 211 & 219 & Car B & HTC 3D \\
\hline
\end{tabular}

Table 1: Traces collected in Hoboken, NJ and Pontiac, MI.

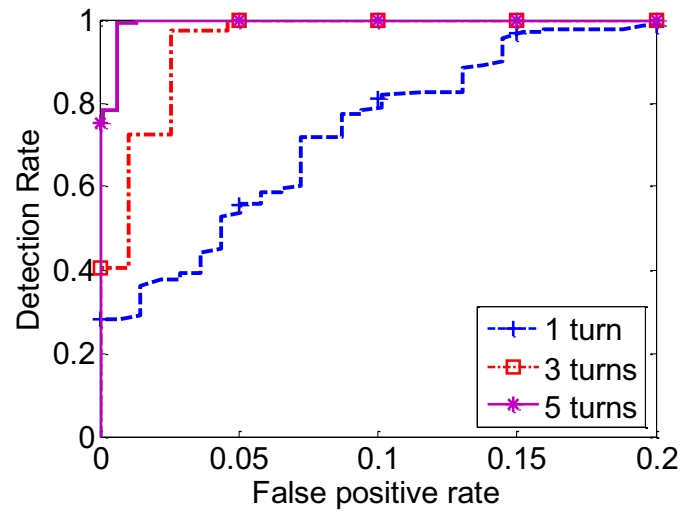

(a) Car A, iPhone4, Hoboken, NJ

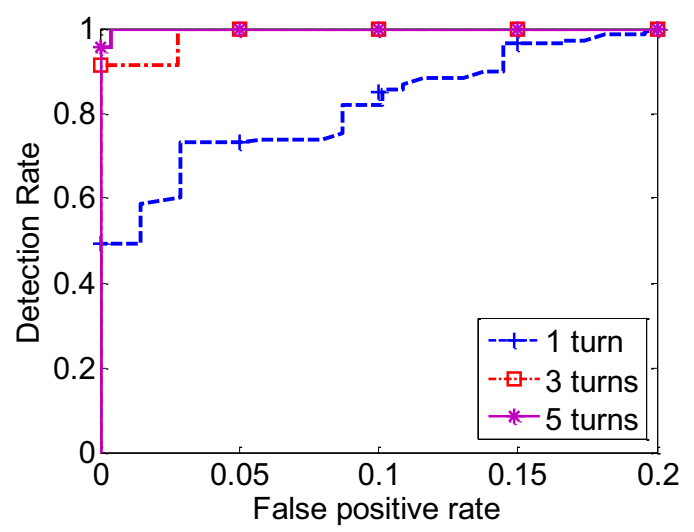

(b) Car B, HTC 3D, Pontiac, MI

Figure 13: ROC curve when using the adapter phone as the reference point.

To evaluate the performance of our system, we define the following metrics:

Accuracy. Accuracy is defined as the percentage of the trials that were correctly classified as driver phone use or passenger phone use.

Detection Rate (DR) and False Positive Rate (FPR). Detection rate is defined as the percentage of driver phone use that are correctly identified by our system, whereas the False positive rate is defined as the percentage of passenger phone use that are classified as driver phone use.

Detection Latency. We define the detection delay as the time needed to make a decision on whether it's a driver phone use or passenger use starting from driving a vehicle.

\subsection{Evaluation Using a Cigarette Lighter Ad- apter}

We evaluate the effectiveness of using the adapter phone placed at the cigarette lighter charger as the reference when driving Car A in Hoboken, NJ and Car B in Pontiac, MI. Figure 13 presents the detection rate versus false positive rate when applying our algorithm to determine the driver phone use. We observe that within one turn, our system achieves over a $80 \%$ detection rate with less than a $10 \%$ false positive rate for both traces in Hoboken and Pontiac. By utilizing multiple turns for detection, the performance is further improved. Specifically, for the experiments in Hoboken, the detection rate goes up to $97 \%$ with a $3 \%$ false positive rate with 3 turns. And with 5 turns, our system can achieve a $99.1 \%$ detection rate with less than a $1 \%$ false positive rate $(0.3 \%$ to be 


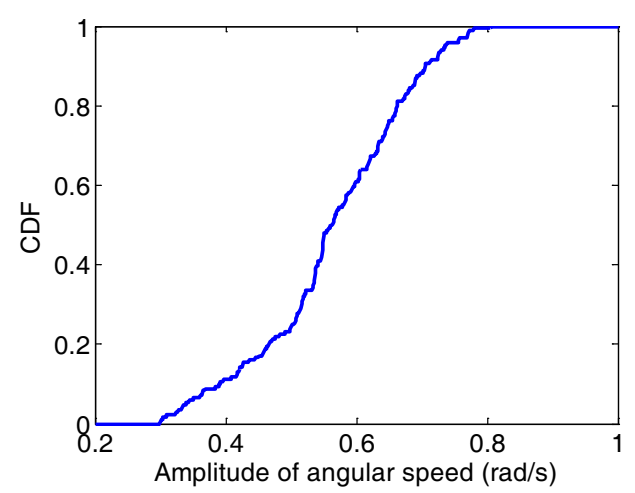

Figure 14: Cumulative distribution function of the angular speed in 570 turns collected from driving in Hoboken, NJ over one month time frame.

exact) in Hoboken. Similarly, for the experiments in Pontiac, we can achieve a $91.4 \%$ detection rate with a $2.4 \%$ false positive rate by using 3 turns, and a $98.42 \%$ detection rate with a $0.92 \%$ false positive rate by using 5 turns. This indicates that our system can achieve very high detection accuracy when the vehicle undergoes only a few turns. We note that the best performance in the ROC curves is achieved when the threshold (for the hypothesis testing as described in Equation (8)) is about $10 \mathrm{~cm}$ away from the center of the car to the passenger side.

\subsection{Evaluation Using an OBD-II Port Adapter}

We further evaluate using the OBD-II speed as reference by applying mixed turns detection, which eliminates the dependence on the bias caused by using the OBD-II.

Filtering Turns with Angular Speed. The noise in the sensing data affects the results of the hypothesis test. Our system adopts a strategy to select turns with a large angular speed so that to get a larger difference of acceleration, thus making our algorithm more robust to noisy sensor readings. As shown in Equation (2), the larger the angular speed is, the more powerful the discrimination becomes in the centripetal acceleration. Given the certain noise level presented in the sensing data, we can thus filter out the turns with small angular speed to improve the detection performance. Our strategy is to choose the turns based on the maximum angular speed and filter out those with the maximum angular speed below a threshold. Figure 14 shows that through our study with 570 turns collected from real-road driving in Hoboken, NJ over one month time period, over $80 \%$ of the turns have maximum angular speed larger than $0.5 \mathrm{rad} / \mathrm{s}$. This suggests that applying our turn selection strategy to cope with the noisy sensing data will only sacrifice a small portion of the data. We thus choose $0.5 \mathrm{rad} / \mathrm{s}$ as the threshold in our study.

Results. Figure 15 presents the system performance when using mixed turns with and without turn selection based on driving traces in Hoboken, NJ. We observe that the performance under turn selection is $20 \%$ better than that without turn selection. In particular, with the turn selection strategy, the detection rate is about $80 \%$ with a false positive rate of $20 \%$ under only 1 set of mixed turns, whereas the detection rate goes up to $91 \%$ with only a $5 \%$ false positive rate based on 3 sets of mixed turns.

\subsection{Evaluation Using Dual Phones}

When there are passengers in the vehicle, our system can leverage a second phone instead of an adapter on the car to determine

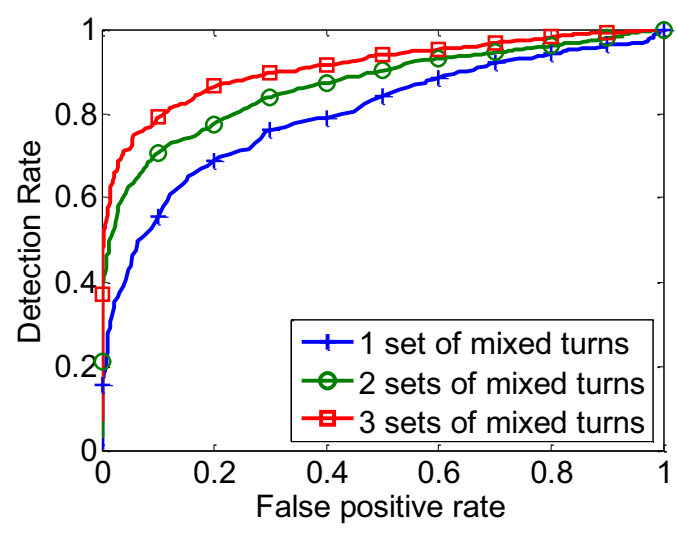

(a) Without turn selection

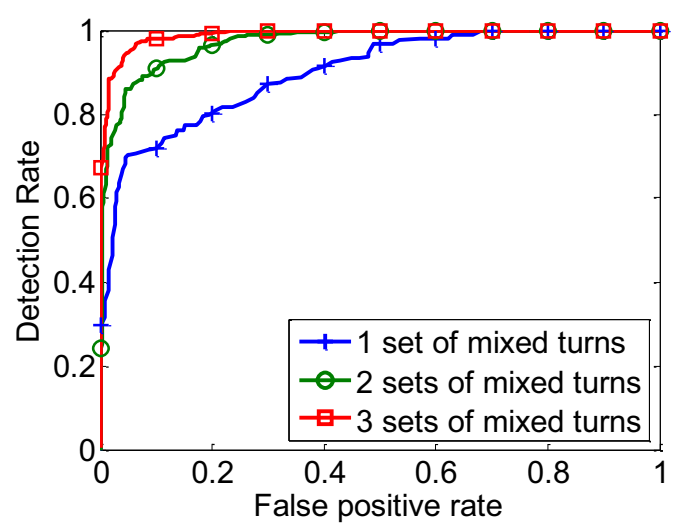

(b) With turn selection

Figure 15: ROC curve when using OBD-II port adapter as the reference point under mixed turns using iPhone4, Car A in Hoboken N.J.

the driver phone. While we have not found any detailed statistics on driver versus passenger cell phone use in vehicles, a federal accident database (FARS) [10] reveals that about $38 \%$ of automobile trips include passengers. Basically, our system can directly compare the centripetal acceleration of these two phones to determine the one on the left side is the driver's phone. These two phones can exchange their centripetal acceleration via Bluetooth. To evaluate such an approach, we carry out a series of experiments by putting one phone at two driver's locations: driver's left pocket (position $A$ ), driver's right pocket (position B), and the other phone at two passenger's locations: passenger's left pocket (position $C$ ), and passenger's right pocket (position $D$ ).

Figure 16 shows the detection accuracy of employing the second phone as reference when driving in two cities when undergoing 1,2 , and 3 turns. We observe that when undergoing one turn the scenario A-D achieves the best detection accuracy, which is over 95\% because the two phones have the largest distance between each other in the vehicle, while the scenario B-C with two phones located in the closest positions achieves about $70 \%$ accuracy under one turn. This is because the significance of the difference of centripetal acceleration between two phones is only affected by the relative distance between them. We find that the accuracy for B-C scenario can go up to $90 \%$ when undergoing 3 turns. We observe similar detection accuracy in Pontiac, for instance, the scenario AD can achieve the detection accuracy over $95.6 \%$ and $99.8 \%$ for 1 and 3 turns respectively. These results show that using the ac- 


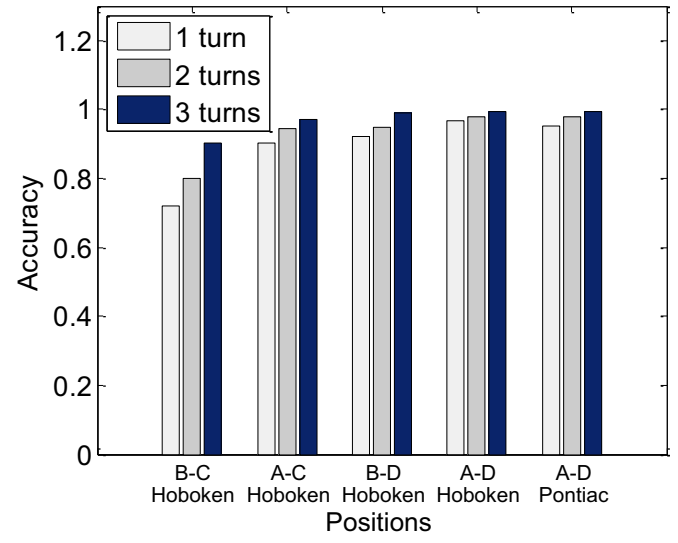

Figure 16: Detection accuracy of using a second phone inside the vehicle as the reference.

celeration from the second phone as the reference generally has good performance in real-world driving tests. Moreover, by using multiple turns, the detection accuracy can be further improved, especially for the phones that are placed very close to each other (i.e. the B-C scenario).

\subsection{Detection Latency}

In reality, it is common that a vehicle experiences more turns at the beginning of a trip before getting onto main roads, such as driving the vehicle out of the parking lot and then driving on local streets. These turns make our system able to determine whether the phone is driver's or passenger's. When sensing driver phone use is conducted using a single turn, the detection latency consists of the algorithm execution time and the turning time (which includes time for sensing data collection). In our system prototype, we find that the algorithm execution time is at the level of sub-millisecond. Thus, the detection latency is determined by the turning time. Based on our experiments summarized in Table 1, the average turning time is about $10 \mathrm{~s}$ in both Hoboken, $\mathrm{NJ}$ and Pontiac, MI.

When multiple turns are employed in our detection algorithm, the time interval between two turns, measured between two maximum angular speeds, dominates the total detection latency. We observe that the average time interval between two turns in Hoboken, NJ is about $28 s$, while it is $18 s$ in Pontiac, MI. Therefore, the latency of our algorithm is the sum of turning times and the time between turns. For instance, when we use two turns with the cigarette lighter adapter, the latency is about $48 s$ and $38 s$ in Hoboken and Pontiac respectively. This indicates that our driver phone sensing algorithm has an acceptable detection latency in environments including both urban and suburban. The time delay in Hoboken city is longer than that in Pontiac. This is because Hoboken has the urban city setting and the driving routes involve more traffic lights and stop signs. Therefore, vehicles experience longer waiting time before making turns.

\section{DISCUSSION}

In this section, we first discuss how this technique can be extended with front-rear detection based on acceleration forces created when the vehicle passes over bumps. We then discuss our initial attempts and results towards a completely phone-based solution, that is a solution that also eliminates the requirement for the plugin adapter. Finally, we speculate about other vehicle sensors

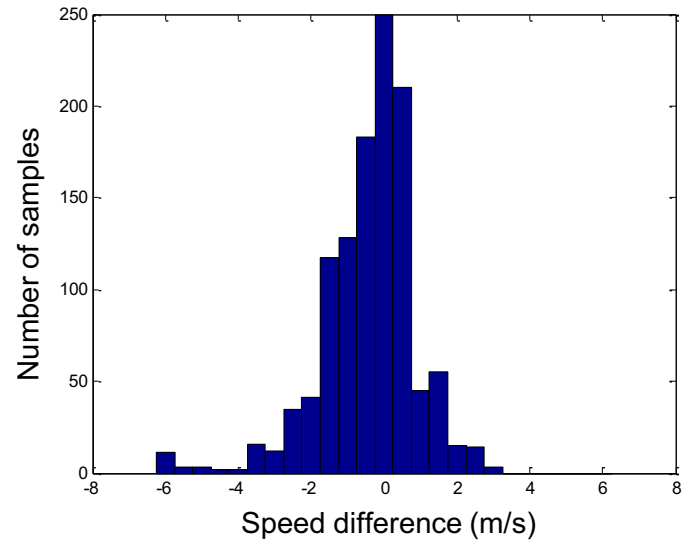

(a) Left door to car's center

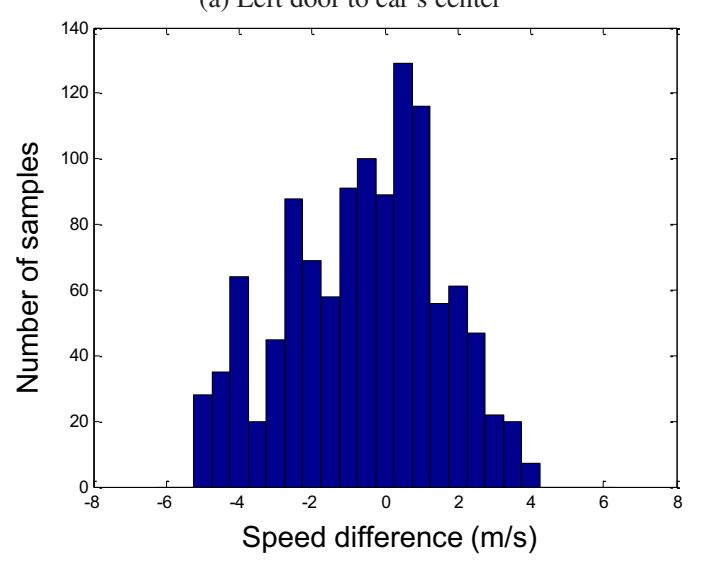

(b) Right door to car's center

Figure 17: Histogram of differential speed between the phone's position on left/right door and at the center of the vehicle.

that could be used as a reference point, when vehicles become a more open platform.

\subsection{Extended System with Front-Rear Detec- tion}

While left-right classification is able to disambiguate the majority of in-vehicle phone use situations, a complete system for driver phone detection involves both left-right and front-rear classifications of phone position. The left-right classification approach proposed in this study can be integrated with the front-rear accelerometer classification described in our previous work [37]. The basic idea of this front-rear classification is that the acceleration forces on a vehicle when passing over speed bumps, potholes, or other uneven surfaces are also position dependent. Consider that the front wheels will hit the bump first, followed by the rear wheels a short time later. Since the front seats are closer to the front wheels, phones at this position will observe a stronger effect from this bump than phones on the rear seats. Our prior experiment show that it could achieve as high as $90 \%$ accuracy when passing two bumps and $94 \%$ when passing three bumps.

\subsection{Towards Infrastructure-Free Driver Phone Use Detection}

One possible infrastructure-free approach is to use the smartphone's GPS speed measurement as a reference. At the first glance, 


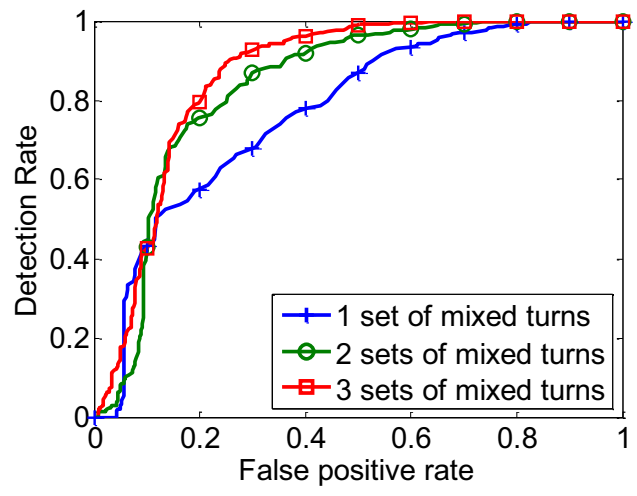

(a) Without turn selection

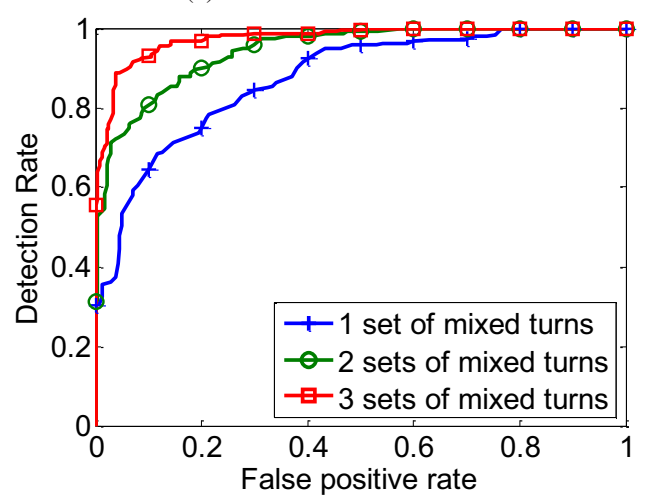

(b) With turn selection

Figure 18: ROC of driver phone use sensing in Hoboken with mixed turns algorithm and GPS speed.

using a speed measurement at the phone position does not seem suitable as a reference. This is less clear however when taking, for example, the GPS chip's internal processing and smoothing into account. Consider a phone on one side and a vehicle moving in a straight line with a constant velocity. In this case, both the instantaneous and smoothed velocities will be the same. When the vehicle turns, the value of the smoothed velocity will lag closer to the center velocity. Assuming the phone's gyroscopes and accelerometers are closer to the instantaneous values, the difference could be used to discriminate the side of the vehicle.

The filters used on GPS chipsets are often proprietary, so we perform experiments to test this hypothesis. We place two iPhone4s on two front doors, and also employ a third iPhone4 in a center cup holder to collect the tangential speed of the center of the vehicle. We then compare the GPS speed from the center phone to those obtained from the front doors. Figure 17 presents the histogram of the differences between the GPS speed of the center phone and the two phones on two front doors. We observe that both histogram of the difference centered at around $0 \mathrm{~m} / \mathrm{s}$, which indicates that the GPS speed is not sensitive to the in-vehicle position, thus making using the smartphone's GPS speed tractable as a replacement of the OBD-II speed.

We further use real road driving experiments to validate the use of phone's GPS. The results presented in Figure 18 and Figure 19 show that for both traces in Hoboken, NJ and Pontiac, MI, our algorithm can achieve over $80 \%$ detection rate with 3 sets of mixed turns without turn selection. Based on turns with $\omega>0.5 \mathrm{rad} / \mathrm{s}$, our algorithm can achieve much better detection rate, that is $90 \%$ and $95 \%$ in Hoboken and Pontiac respectively. These results show

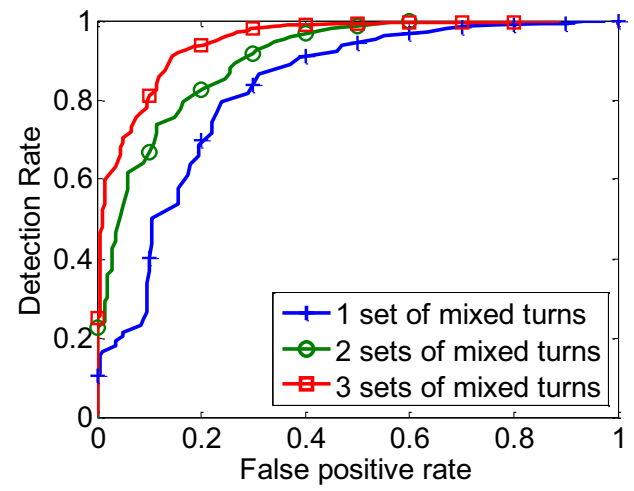

(a) Without turn selection

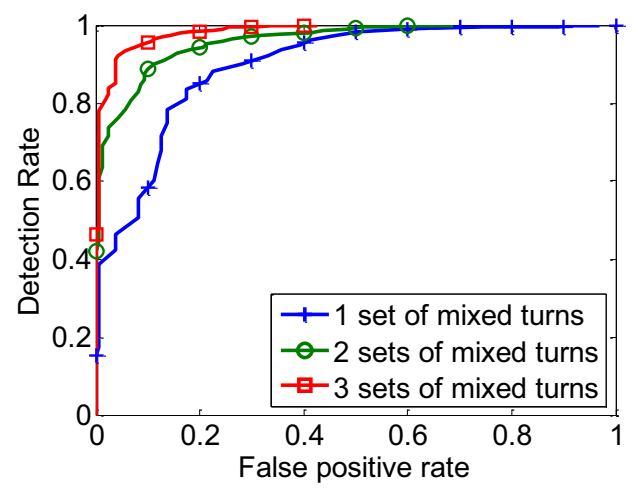

(b) With turn selection

Figure 19: ROC of driver phone use sensing in Pontiac with mixed turns algorithm and GPS speed.

some promise in using the GPS speed measured by the phone to derive the vehicle reference and possible achieve completely infrastructure-free driver detection. While cannot fully explain these observations, we believe these results warrant further study.

\subsection{Integration with Additional Vehicle Sen- sors}

Our work also points to a more intriguing possibility of vehicular smartphone applications where all the vehicle's sensors are available to an authorized smartphone. While ODB-II to Bluetooth is a first step in this direction, much richer interfaces with additional information are possible and have been realized in select vehicles. For example, the Open-XC interfaces [13] provides additional vehicle parameters to Android phones. Of particular interest, is the steering wheel angle measured by a steering wheel position sensor [6] (this sensor normal provides information for electronic stability control). Having such information available would provide additional and potentially more accurate means for determining the turn radius of the vehicle and estimating acceleration forces at a vehicle reference point.

\section{CONCLUSION}

In this paper we demonstrate a low-infrastructure approach for discriminating between a phone in the driver or passenger position of a moving vehicle by sensing vehicle dynamics. It does not rely on a built-in handsfree Bluetooth system in the car but only on the phone's embedded sensors and a simple plug-in reference module for the cigarette lighter or OBD-II port. The insight that the cen- 
tripetal acceleration varies depending on the position in the car enables us to build a system that exploits the difference of centripetal acceleration at different positions inside the vehicle to determine the driver phone when turning. Our system accomplishes the task by comparing the measured centripetal acceleration at the phone with that from a reference point in the vehicle. Instead of such a reference point, the system could also leverage a second phone in the car to perform detection when available.

We demonstrate the generality of our approach through extensive experiments with two different phone types and two different cars in two cities over a month-long time period. Our findings show that our approach yields close to $100 \%$ accuracy using only a few turns with less than $3 \%$ false positive rate. While the system has to wait until the vehicle has passed through one or more turns, our experiments show that detection is often possible by the time a vehicle leaves a parking lot or before it reaches a main road, so the determination is available for the vast majority of trips.

\section{ACKNOWLEDGEMENT}

This work is supported in part by the National Science Foundation Grants CNS-0954020, CNS-1016303, CNS-1040735 and CNS-0845896.

\section{REFERENCES}

[1] Android Developers. http://developer.android.com/reference/android /hardware/SensorEvent.html.

[2] AT\&T chief speaks out on texting at the wheel. http://www.nytimes.com/2012/09/20/technology/att-chiefspeaks-out-on-texting-while-driving.html.

[3] AT\&T driver safety app. http://www.theverge.com/2012/8/15/3243963/att-textingdriving-safety-app.

[4] Distracted driving and driver, roadway, and environmental factors. http://www.distraction.gov/download/researchpdf/Distracted-Driving-and-Driver-Roadway-EnvironmentalFactors.pdf.

[5] Drivesmart plus. http://tinyurl.com/4v7oygy.

[6] Electronic stability control. http://en.wikipedia.org/wiki/Electronic_stability_control.

[7] Guardian angel vehicle platform. http://www.trinitynoble.com/.

[8] InvenSense MPU-6000/6050 Six-Axis (Gyro + Accelerometer) MEMS Motion Tracking Devices. http://www.invensense.com/mems/gyro/mpu6050.html.

[9] Key2safedriving app. http://www.key2safedriving.com/.

[10] National highway traffic safety administration:Fatality analysis reporting system. http://tinyurl.com/24h2t7.

[11] No call, no text, no update behind the wheel: NTSB calls for nationwide ban on PEDs while driving. http://www.ntsb.gov/news/2011/111213.html.

[12] Textecution. http://www.textecution.com/.

[13] The OpenXC Platform. http://openxcplatform.com/.

[14] Txtblocker. http://www.txtblocker.com/.

[15] G. Chandrasekaran and et.al. Tracking vehicular speed variations by warping mobile phone signal strengths. In IEEE PerCom, 2011.

[16] H. Chu, V. Raman, J. Shen, R. Choudhury, A. Kansal, and V. Bahl. Poster: You driving? talk to you later. In $A C M$ MobiSys, 2011.
[17] J. Dai, J. Teng, X. Bai, Z. Shen, and D. Xuan. Mobile phone based drunk driving detection. In PervasiveHealth, 2010.

[18] H. Eren, S. Makinist, E. Akin, and A. Yilmaz. Estimating driving behavior by a smartphone. In Proceedings of IEEE Intelligent Vehicles Symposium (IV), 2012.

[19] J. Eriksson, L. Girod, B. Hull, R. Newton, S. Madden, and H. Balakrishnan. The pothole patrol: Using a mobile sensor network for road surface monitoring. In ACM MobiSys, 2008.

[20] M. Fazeen, B. Gozick, R. Dantu, M. Bhukhiya, and M. C. Gonzalez. Safe driving using mobile phones. IEEE Transactions on Intelligent Transportation Systems (TITS), 2012.

[21] J. A. Geen. Very low cost gyroscopes. In IEEE Sensors, 2005.

[22] A. Glassner. Graphics Gems. Morgan Kaufmann, 1990.

[23] D. Gundlegard and J. Karlsson. Handover location accuracy for travel time estimation in GSM and UMTS. In IEEE ITSC, 2009.

[24] D. Johnson and M. Trivedi. Driving style recognition using a smartphone as a sensor platform. In Proceedings of the 14th IEEE International Conference on Intelligent Transportation Systems (ITSC), 2011.

[25] K. A. Li, P. Baudisch, and K. Hinckley. Blindsight: Eyes-free access to mobile phones. In ACM CHI, 2008.

[26] J. Lindqvist and J. Hong. Undistracted driving: A mobile phone that doesn't distract. In HotMobile, 2011.

[27] H. Liu, Y. Gan, J. Yang, S. Sidhom, Y. Wang, Y. Chen, and F. Ye. Push the limit of wifi based localization for smartphones. In ACM Mobicom, 2012.

[28] A. Mednis, G. Strazdins, R. Zviedris, G. Kanonirs, and L. Selavo. Real time pothole detection using android smartphones with accelerometers. In IEEE DCOSS, 2011.

[29] P. Mohan, V. Padmanabhan, and R. Ramjee. Nericell: Rich monitoring of road and traffic conditions using mobile smartphones. In ACM SenSys, 2008.

[30] M. Mun and et.al. PEIR, the personal environmental impact report, as a platform for participatory sensing systems research. In ACM MobiSys, 2009.

[31] L. Nelson, S. Bly, and T. Sokoler. Quiet calls: Talking silently on mobile phones. In ACM CHI, 2001.

[32] R. Serway and J. Jewett. Physics for scientists and engineers. Brooks/Cole Publishing Company, 2009.

[33] A. Thiagarajan, J. Biagioni, T. Gerlich, and J. Eriksson. Cooperative transit tracking using smart-phones. In $A C M$ SenSys, 2010.

[34] J. White, C. Thompson, H. Turner, B. Dougherty, and D. C. Schmidt. WreckWatch: Automatic traffic accident detection and notification with smartphones. Mobile Networks and Applications, 2011.

[35] M. Wiberg and S. Whittaker. Managing availability: Supporting lightweight negotiations to handle interruptions. ACM Transactions on Computer-Human Interaction, 2005.

[36] J. Yang, S. Sidhom, G. Chandrasekaran, T. Vu, H. Liu, N. Cecan, Y. Chen, M. Gruteser, and R. Martin. Detecting driver phone use leveraging car speakers. In ACM MobiCom, 2011.

[37] J. Yang, S. Sidhom, G. Chandrasekaran, T. Vu, H. Liu, N. Cecan, Y. Chen, M. Gruteser, and R. Martin. Sensing driver phone use with acoustic ranging through car speakers. IEEE Transactions on Mobile Computing (TMC), 2012. 